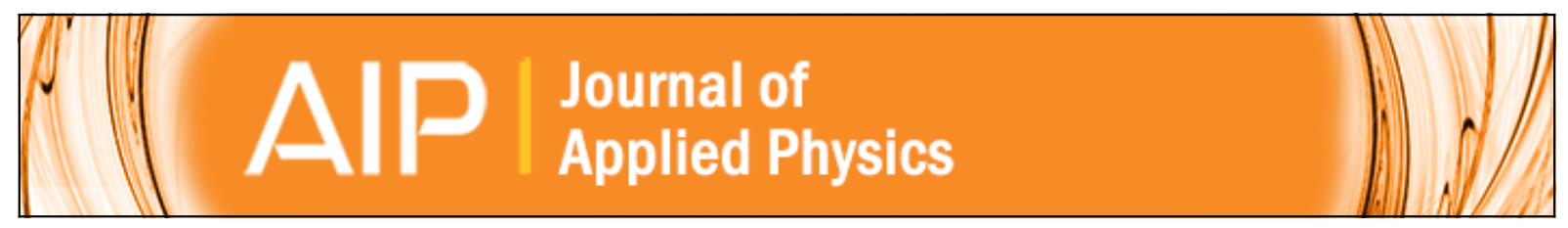

\title{
Magnetotransport in NiMnGa thin films
}

Andriy Vovk, Leszek Malkinski, Vladimir Golub, Charles O’Connor, Zhenjun Wang, and Jinke Tang

Citation: Journal of Applied Physics 97, $10 \mathrm{C503}$ (2005); doi: 10.1063/1.1847411

View online: http://dx.doi.org/10.1063/1.1847411

View Table of Contents: http://scitation.aip.org/content/aip/journal/jap/97/10?ver=pdfcov

Published by the AIP Publishing

\section{Articles you may be interested in}

Ferromagnetic resonance properties and anisotropy of Ni-Mn-Ga thin films of different thicknesses deposited on Si substrate

J. Appl. Phys. 105, 07 A942 (2009); 10.1063/1.3075395

Structural transition of ferromagnetic Ni $2 \mathrm{MnGa}$ nanoparticles

J. Appl. Phys. 101, 063530 (2007); 10.1063/1.2713370

Anomalous magnetoresistance in NiMnGa thin films

J. Appl. Phys. 96, 3865 (2004); 10.1063/1.1771474

Ferromagnetic resonance in Ni-Mn-Ga films

Appl. Phys. Lett. 81, 1279 (2002); 10.1063/1.1501161

$\mathrm{Ni}-\mathrm{Mn}-\mathrm{Ga}$ thin films produced by pulsed laser deposition

J. Appl. Phys. 91, 8234 (2002); 10.1063/1.1452222
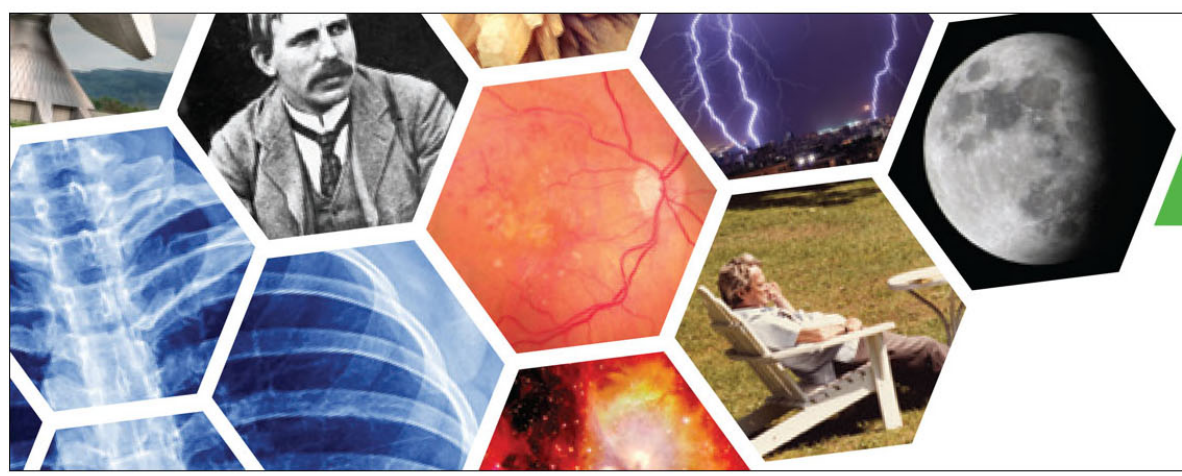

\section{SUBSCRIBE TO




\title{
Magnetotransport in NiMnGa thin films
}

\author{
Andriy Vovk, ${ }^{\text {a) }}$ Leszek Malkinski, Vladimir Golub, ${ }^{\text {b) }}$ and Charles O'Connor \\ Advanced Materials Research Institute, University of New Orleans, 2000 Lakeshore Drive, New Orleans, \\ Louisiana 70148 \\ Zhenjun Wang and Jinke Tang \\ Department of Physics, University of New Orleans, 2000 Lakeshore Drive, New Orleans, Louisiana 70148
}

(Presented on 9 November 2004; published online 4 May 2005)

\begin{abstract}
The influence of substrate temperature and annealing conditions on structure, magnetic, and magnetotransport properties of NiMnGa films was investigated, and the crucial effect of substrate temperature was confirmed. It was found that amorphous disordered films are formed during deposition on a substrate held at room temperature. Postdeposition annealing leads to partial recrystallization and recovery of magnetic properties. Annealing at $773 \mathrm{~K}$ does not allow the formation of crystal structure, which is created during deposition on substrates held at the same temperature. The highest values of magnetoresistance were observed in the films with a well-defined crystal structure. (C) 2005 American Institute of Physics. [DOI: 10.1063/1.1847411]
\end{abstract}

$\mathrm{Ni}_{2} \mathrm{MnGa}$ alloys are magnetic shape memory systems that show large magnetic-field-induced strain up to $10 \% .^{1}$ These materials are considered to be promising for magnetic actuator devices. Another interesting feature that has recently been discovered ${ }^{2-6}$ is the negative magnetoresistance effect, which occurs for NiMnGa and some other Heusler alloys. The magnetoresistance value varies in the range from $0.5 \%$ to $5 \%$ depending on the composition of the films and preparation technique. In a previous study, ${ }^{6}$ it was shown that films of NiMnGa deposited by pulsed laser deposition (PLD) show values of magnetoresistance (MR) as high as 5\% in the field of $50 \mathrm{kOe}$ at $5 \mathrm{~K}$ and $\sim 3 \%$ at room temperature (RT). These values are the highest reported to date for the thin NiMnGa films. It was also shown that it is possible to create a material with a linear dependence of MR versus magnetic field $(H)$ over a wide temperature range. This feature opens a possibility for the development of a magnetic-field sensor material with a very small temperature variation of resistance and magnetoresistance.

It is known that the structure and properties of Heusler alloy films strongly depend on the details of the preparation procedure. ${ }^{7}$ In this work, we study the influence of the substrate temperature and postdeposition annealing on the structure and magnetotransport properties of NiMnGa films.

The films were deposited on $\mathrm{Al}_{2} \mathrm{O}_{3}$ substrates held at RT and at $773 \mathrm{~K}$ by pulsed laser deposition technique described elsewhere. ${ }^{6}$ The films deposited at RT were annealed in Ar $+\mathrm{H}_{2}(5$ at. \%) at temperatures up to $773 \mathrm{~K}$. The composition of the target used for depositions was $\mathrm{Ni}_{52.3} \mathrm{Mn}_{27.4} \mathrm{Ga}_{20.3}$ and the composition of the film was determined by energy dispersive X-ray analysis (EDAX) - $\mathrm{Ni}_{53} \mathrm{Mn}_{24} \mathrm{Ga}_{23}$. The structure of the films was studied using field-emission scanning electron microscope (FESEM, model LEO 1530VP) and X-ray diffractometry ( $\mathrm{Cu} K \alpha$ radiation). Magnetotransport

\footnotetext{
a) On leave from the Institute of Magnetism NAS of Ukraine, 36b Vernadskogo Blvd., 03142 Kiev, Ukraine; electronic mail: avovk@uno.edu

${ }^{b)}$ On leave from the Institute of Magnetism NAS of Ukraine, 36b Vernadskogo Blvd., 03142 Kiev, Ukraine.
}

measurements were carried out using Quantum Design PPMS model 6000 in the $5-350-\mathrm{K}$ temperature range and in the fields of up to $90 \mathrm{kOe}$. MR was measured using a fourpoint technique in current in-plane configuration. The magnetic field was applied in the film plane and perpendicular to the current ( $T$ geometry). Ferromagnetic resonance was studied at $300 \mathrm{~K}$ using an $X$-band Bruker EMX300 electronparamagnetic-resonance (EPR) spectrometer.

The structure of NiMnGa alloys is very sensitive to their composition and preparation technique. The x-ray investigations (data not shown) demonstrate that the films deposited on a substrate held at $773 \mathrm{~K}$ contain a cubic austenite phase $(a \approx 0.581 \mathrm{~nm})$, which is very close to the $a=0.582 \mathrm{~nm} \mathrm{ob}-$ served in bulk materials, some tetragonal martensite and also residual MnO. ${ }^{6}$ FESEM investigations confirm that the films are polycrystalline with an average grain size of $\sim 70 \mathrm{~nm}$ (see Fig. 1). On the contrary, the films deposited on a substrate held at RT are considerably disordered. The diffraction pattern shows only a broad halo typical for nanocrystalline (almost amorphous) alloy. FESEM also shows no specific features. Annealing of the films deposited at RT at $773 \mathrm{~K}$ leads to recrystallization. After annealing, a broad peak in the vicinity of the strongest line $\langle 220\rangle$ of cubic austenite phase appears. At the same time FESEM shows the formation of crystallites with the size ranging from 20 to $50 \mathrm{~nm}$ embedded in a quasiamorphous matrix [Fig. 1(a)]. It is obvious that the annealing temperature of $773 \mathrm{~K}$ is not high enough to cause the formation of the structure similar to that observed for the films deposited on a substrate held at $773 \mathrm{~K}$.

Ferromagnetic resonance (FMR) is a powerful tool to study the magnetic properties of the films. Previous studies ${ }^{6,8}$ on NiMnGa films show that it is possible to determine the details of magnetic homogeneity and martensite-austenite transition. In our case, FMR measured at room temperature for the films deposited on a substrate held at $773 \mathrm{~K}$ shows the presence of two magnetic phases with a difference in saturation magnetizations of several percent. This confirms the data of x-ray investigations that the film contains a mix- 

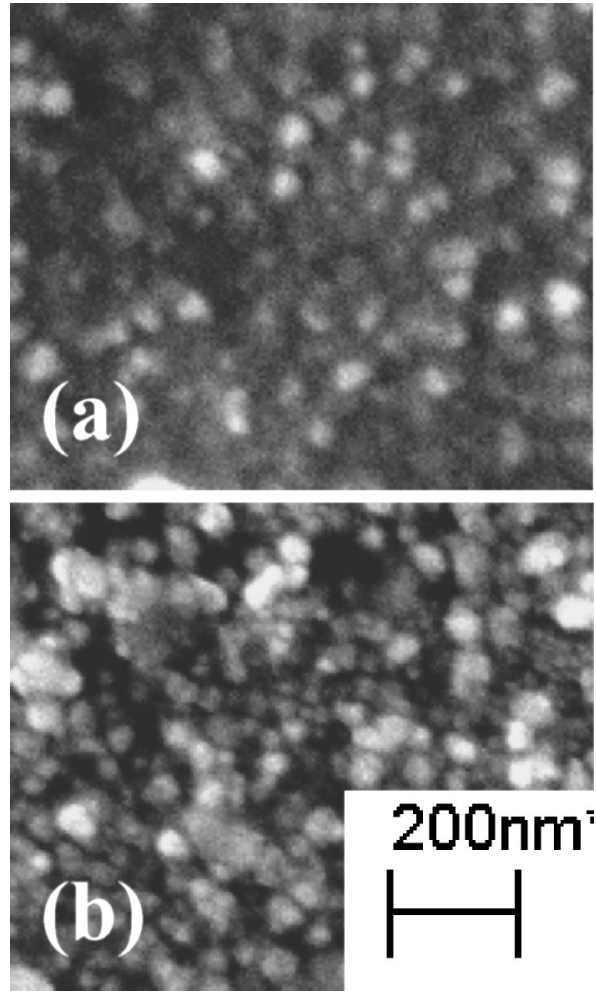

FIG. 1. FESEM images of the films deposited on a substrate held at RT and annealed at $773 \mathrm{~K}$ (a) and deposited on a substrate held at $773 \mathrm{~K}$ (b).

ture of austenite and martensite phases. ${ }^{6}$ The film deposited on a substrate held at RT is nonmagnetic. No resonance peak is observed for the fields $\sim 6500$ Oe. A weak peak appears near 3500 Oe. It is characteristic of a paramagnetic phase. Unfortunately, the detailed analysis of this peak is not possible as it interferes with the strong signal of the substrate. After annealing a recovery of magnetic properties occurs. One can find a broad FMR spectrum with a line center near the position of the austenite line for the films deposited on a substrate held at $773 \mathrm{~K}$ (Fig. 2). The width of the line shows high inhomogeneity of the film.

The temperature dependencies of resistance $(R)$ for the films have metallic characteristics in the whole temperature $(T)$ range. However, the film deposited on a substrate held at RT demonstrates a vanishing variation of $R$ vs $T$ (Fig. 3).

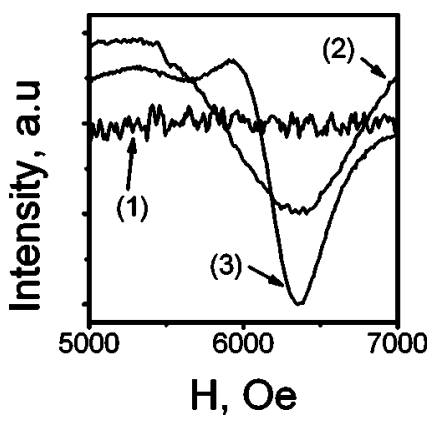

FIG. 2. FMR spectra for the films deposited on a substrate held at RT before (Ref. 1) and after annealing at $773 \mathrm{~K}$ (Ref. 2), and deposited on a substrate held at $773 \mathrm{~K}$ (Ref. 3) measured at $300 \mathrm{~K}$. The magnetic field is perpendicular to the film plane. The high-field peak corresponds to martensite phase, the low-field one - to austenite.

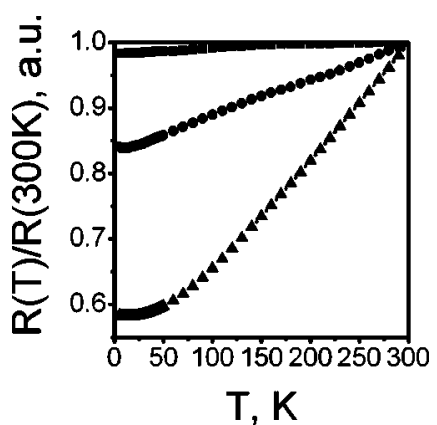

FIG. 3. The dependences of the $R$ vs $T$ for the films deposited on a substrate held at RT before (solid square) and after annealing at $773 \mathrm{~K}$ (solid circle) and deposited on a substrate held at $773 \mathrm{~K}$ (solid triangle).

The value of $R(5 \mathrm{~K}) / R(300 \mathrm{~K})$ is only $\sim 0.98$. Annealing at $773 \mathrm{~K}$ leads to an increase of temperature variation of $R$ and the value becomes $\sim 0.84$. The highest relative variation $R$ vs $T(\sim 0.58)$ was observed for the film deposited on a substrate held at $773 \mathrm{~K}$. A small $R$ vs $T$ variation is typical for disordered alloys. ${ }^{9}$ The temperature dependence of resistance confirms the fact that the film deposited on a cold substrate has a highly disordered structure. The martensite-austenite transition does not lead to pronounced peculiarities of $R$ vs $T$ dependences, as in the case of NiMnGa bulk alloys, ${ }^{10}$ due to the expanded character of the transformation. The discussion on this matter can be found in Ref. 6.

Comparing the data with the structure, FMR, and $R$ vs $T$ measurements, one can conclude that substrate temperature has a crucial effect on the film's structure and transport properties. During deposition on a substrate held at RT, highly disordered alloys are formed. Unlike the bulk and the crystalline film deposited on a substrate held at $773 \mathrm{~K}$, the amorphous alloy films are not ferromagnetically ordered. Annealing of such an amorphous film partially restores its crystalline structure and magnetic properties. A significant structural dependence of the magnetic properties of Heusler alloys is a well-known phenomenon. ${ }^{10,11}$ Also, improper occupation of the atom sites in the lattice, or atomic disorder for the semi-Heusler NiMnSb alloy, ${ }^{12}$ leads to a decrease in spin polarization, which explains the structural dependence of the magnetic and transport properties.

In Fig. 4 typical dependencies of MR versus $H$ are shown. It is worthwhile to note that the film deposited on a substrate held at RT shows negligible values of MR in the

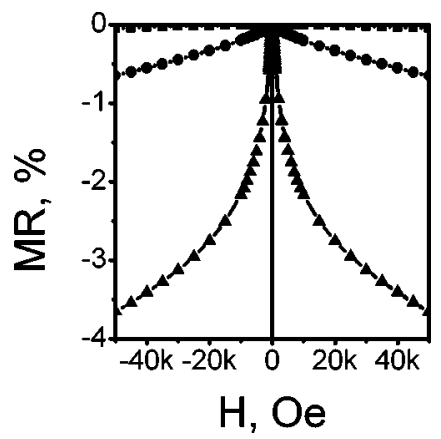

FIG. 4. MR vs $H$ dependencies measured at $100 \mathrm{~K}$ for the films deposited on a substrate held at RT after annealing at 573 (solid square) and $773 \mathrm{~K}$ (solid circle) and deposited on a substrate held at $773 \mathrm{~K}$ (solid triangle). 
whole investigated temperature range. A small MR effect was first observed at low temperatures after annealing at 573 $\mathrm{K}$. The values of MR continue to increase with annealing temperature but do not reach the ones for the films deposited on hot substrates. Also, after annealing, the values of MR measured at RT do not exceed $0.5 \%$. Thus, due to structure and magnetic properties, the MR effect is strongly suppressed for amorphous NiMnGa films. Annealing at the higher temperatures leads to recrystallization and restoration of the magnetic properties of the alloys. From this sizable MR appears. However, according to our data, annealing does not allow the film to obtain the same structure, magnetic state, and values of MR as for the films deposited on hot substrates. It is obvious that deposition on a substrate held at $773 \mathrm{~K}$ allows the formation of a crystal structure with better magnetic properties and higher values of MR.

In conclusion, we studied the influence of substrate temperature and postdeposition annealing on the magnetotransport properties of NiMnGa films. It was shown that the magnetotransport properties of the films strongly depend on the crystalline structure that is formed during deposition. The temperature of the substrate during depositions has a greater effect on the structure, magnetic, and magnetotransport properties of the films than the temperature of the postdeposition annealing. The highest values of MR were found for the magnetic films with a well-defined crystal structure, i.e., deposited on substrates held at high temperature. Additional experiments should be made to improve the technology, which would allow developing materials with high values of MR suitable for sensor applications.

We thank Dr. Alexei Sozinov for stimulating discussion and help with the target preparation and structure interpretation. We are grateful to Dr. W. Zhou for the help with FESEM investigations. This work was supported by DoD/ DARPA Grant No. MDA972-04-1-0001.

${ }^{1}$ A. Sozinov, A. A. Likhachev, N. Lanska, and K. Ullakko, Appl. Phys. Lett. 80, 1746 (2002)

${ }^{2}$ P. G. Tello, F. J. Castaño, R. C. O'Handley, S. M. Allen, M. Esteve, F. Castaño, A. Labarta, and X. Batlle, J. Appl. Phys. 91, 8234 (2002).

${ }^{3}$ M. S. Lund, J. W. Dong, J. Lu, X. Y. Dong, C. J. Palmstrøm, and C. Leighton, Appl. Phys. Lett. 80, 4798 (2002).

${ }^{4}$ S. J. Lee, Y. P. Lee, Y. H. Hyun, and Y. V. Kudryavtsev, J. Appl. Phys. 93, 6975 (2003).

${ }^{5}$ J. Marcos, A. Planes, L. Mañosa, A. Labarta, and B. J. Hattink, Phys. Rev. B 66, 054428 (2002).

${ }^{6}$ V. O. Golub, A. Ya. Vovk, L. Malkinski, C. J. O'Connor, Zh. Wang, and J. Tang, J. Appl. Phys. 96, 3865 (2004).

${ }^{7}$ Yu. V. Kudryavtsev, Y. P. Lee, and J. Y. Rhee, Phys. Rev. B 66, 115114 (2002)

${ }^{8}$ S. I. Patil et al., Appl. Phys. Lett. 81, 1279 (2002).

${ }^{9}$ P. A. Lee and T. V. Ramakrishnan, Rev. Mod. Phys. 57, 287 (1985).

${ }^{10}$ V. O. Golub, A. Ya. Vovk, C. J. O'Connor, V. V. Kotov, P. G. Yakovenko, and K. Ullakko, J. Appl. Phys. 93, 8504 (2003).

${ }^{11}$ C. J. O'Connor, V. O. Golub, A. Ya. Vovk, V. V. Kotov, P. G. Yakovenko, and K. Ullakko, IEEE Trans. Magn. 38, 2844 (2002).

${ }^{12}$ D. Orgassa, H. Fujiwara, T. C. Schulthess, and W. H. Butler, Phys. Rev. B 60, 13237 (1999). 